\title{
Triple-Helix Structured Model Based on Problem-Knowledge-Solution Co-evolution for Innovative Product Design Process
}

\author{
Qian Hui ${ }^{1,2}$, Yan Li ${ }^{1,2^{*}} \mathbb{D}$, Ye Tao ${ }^{1,2}$ and Hongwei Liu ${ }^{1,2}$
}

\begin{abstract}
A design problem with deficient information is generally described as wicked or ill-defined. The information insufficiency leaves designers with loose settings, free environments, and a lack of strict boundaries, which provides them with more opportunities to facilitate innovation. Therefore, to capture the opportunity behind the uncertainty of a design problem, this study models an innovative design as a composite solving process, where the problem is clarified and resolved from fuzziness to satisfying solutions by interplay among design problems, knowledge, and solutions. Additionally, a triple-helix structured model for the innovative product design process is proposed based on the co-evolution of the problem, solution, and knowledge spaces, to provide designers with a distinct design strategy and method for innovative design. The three spaces interact and co-evolve through iterative mappings, including problem structuring, knowledge expansion, and solution generation. The mappings carry the information processing and decision-making activities of the design, and create the path to satisfying solutions. Finally, a case study of a reactor coolant flow distribution device is presented to demonstrate the practicability of this model and the method for innovative product design.
\end{abstract}

Keywords: Innovative design, Problem-knowledge-solution co-evolution, Design activity, Design process, Product design

\section{Introduction}

A design problem is usually described negatively as illdefined or wicked because of its vague initial state, either unknown or ambiguous goal state, and insufficient information [1]. Nevertheless, in this study, it is believed that these characteristics are not so much the downside of the problem but conversely make a design problem innovative. Owing to information insufficiency, designers are left with loose settings, free environment, and less strict boundaries to explore creative ideas from the problem uncertainty.

Design is highly underdetermined from both framing and solution-seeking perspectives, and appears as

\footnotetext{
${ }^{*}$ Correspondence: liyan@scu.edu.cn

${ }^{1}$ Innovation Method and Creative Design Key Laboratory of Sichuan

Province, Chengdu 610065, China

Full list of author information is available at the end of the article
}

a process of decision making under uncertainty [2]. To fully explore and create propositions on design objects with desirable and unknown statements, the design process is modeled as an iterative synthesis cycle. The innovative problem is re-analyzed multiple times as new changes are included in the design, which act as potential or essential opportunities for designers to identify the problem [3]. It is believed that iteration is one of the most basic features of design $[4,5]$. In some studies $[6,7]$, iterative design cycles are modeled as transitions between information processing activities and decision making, and innovative design involves more frequent iterations among the activities of problem analysis, idea generation, composition of ideas from other ideas, and evaluation. Product development is not only a process of seeking solutions, but also involves problem exploration and effective utilization of knowledge [8]. Therefore, the understanding and solving of a design problem is both 
solution- and knowledge-dependent. Innovative design is a process of problem refining, during which the problem is represented by different granularity, and the knowledge at the different granularity levels is transferred and adopted in the target domain to inspire creative ideas [9, 10]. Moreover, the generated solutions stimulate designers to re-think the definition of the problem. Therefore, iterations of the innovative design should comprise a series of design activities involving the problem, knowledge, and solution.

Therefore, to further explore the iterative process of innovative design, this paper reports a distinct design strategy and method for innovative design, and models innovative design as a composite process of problem (re-)framing, knowledge creation, and solution generation. With the help of the proposed strategy and model, the designer can better understand the solving process of innovative design, and clarify and resolve the design problem from fuzziness to satisfying solutions. This study not only provides theoretical support for further research in innovative design, but also lays a foundation for the construction of computer-aided innovative design systems.

\section{Related Works}

Over the past few decades, considerable work has been published on the design process. One of the viewpoints formalizes the design process as a search process when the goals of the design are well-defined before a search commences, and the focus of the design is not changed until a solution is found $[11,12]$. The search begins with a problem state and proceeds through intermediate design states until the goal state is reached, and the problem is solved $[13,14]$. In addition to the search process, design may also be viewed as an exploration when the focus of the design changes as the process continues [15]. Because a design problem is now well-known as an ill-defined or wicked problem, with a vague initial state, either unknown or ambiguous goal state, and insufficient information [4], it cannot be understood and defined once and for all. During design, the actions taken in the problem space (e.g., problem structuring) are influenced by those taken in the solution space (e.g., idea generation) and vice versa [16]. Therefore, design problems are considered to evolve alongside the solutions.

To solve the ill-defined problem, some researchers [17-19] put forward that a designer engages in an initial problem structuring phase before moving into solution development. This cognitive strategy is modeled as problem-driven, through which the designer can gain a thorough understanding of the problem, and a highly defined problem space at the expense of generating more ideas. Additionally, in other studies [20-23], it was observed that some designers preferred to jump into the solution space before the problem was formulated. This strategy is formalized as solution-driven, through which the designer can move rapidly to early solution conjectures and take the conjectures as a way of exploring and defining both problems and solutions.

Moreover, in contrast to the two phase-based views, arguably a most noteworthy idea in recent years describes design as a process where design problems and potential solutions co-evolve over time. Mary Lou Maher first proposed the co-evolution model of design as involving interactions between the problem and solution spaces $[12,15,24,25]$. A simple genetic algorithm was assumed as the basis for co-evolution that the definition of a problem could change in response to the current status of the solution space as opposed to being fixed and defined once-and-for-all. The interactions between these two spaces can bring new variables into both spaces, causing unexpected design requirements or solutions.

Based on the refinement of Maher's original model, Dorst and Cross [26] then put forward a co-evolution model (see Figure 1) to apply Maher's computational concept to a behavioral analysis of human creativity in design [27]. In the revised co-evolution model, it was suggested that the creative event is not so much a "creative leap" from the problem to the solution whereby the designer first fixes the problem and then searches for a solution; it occurs when a problem-solution pairing is framed, during which the designer forms coherent information and helps to crystallize core solution ideas. By adopting this model, the ill-defined or wicked problem is solved with the integration of the solution, and both the problem formulation and solution ideas are developed and refined by a constant iteration of analysis, synthesis, and evaluation between the two spaces.

The iteration between the problem and solution spaces in the co-evolution model provides a mechanism for

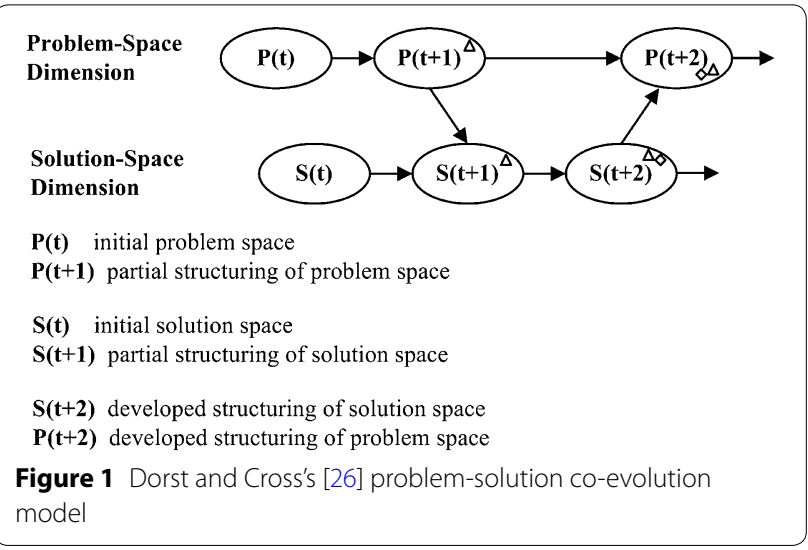


transferring knowledge between the two spaces, with the potential of expanding their boundaries during the design process. However, research on the problem, the solution co-evolution model mainly focused on the design problem and solution, but paid little attention to design knowledge. The knowledge flows alongside the entire design process, and also evolves as changes are involved in design. It is critical to both problem understanding and solution generation. Therefore, it is necessary to characterize the knowledge as another key to design, and further discuss its effect on the interplay between problem formulation and solution ideas.

Furthermore, another design approach with iterative design actions, referred to as the C-K theory, was introduced by Hatchuel and Weil $[28,29]$. Unlike the previous design methods, the $\mathrm{C}-\mathrm{K}$ theory emphasizes that something unknown can intentionally emerge from known knowledge, which is contradictory to a well-structured theory. It is pointed out in this theory that a formal distinction between spaces of "Concepts" (C) and "Knowledge" (K) is a condition for design. Described in the $\mathrm{C}-\mathrm{K}$ theory, design is modeled using four operations (C-C, C-K, K-C, C-C), where a concept generates other concepts or is transformed into knowledge, and new knowledge is created in favor of further pursuit of the undecided concepts [30-33]. Illustrated in Figure 2, creative concepts are the potential "seeds" for design alternatives, and they are undecided with respect to the existing knowledge at the point of time when it is conceived. If this concept is pursued further, such as by adding, removing, or varying some attributes, it expands from the original one and can replace it, while new knowledge evolves in favor of the advanced concept. Consequently, both the evolved knowledge and conceived concept become part of design, the creative concept is adopted as the design solution and becomes new knowledge.

The C-K theory is built as an original formalism of the design reasoning used in innovation. Nevertheless, its contribution to the design process by guidance and assistance is unclear [30,35]. In the $\mathrm{C}-\mathrm{K}$ theory, there is a lack of criteria that allows the designer to decide on the next course of action at a given time. In this case, by using the $\mathrm{C}-\mathrm{K}$ theory, the designer cannot decide the order in which properties are related to an initial concept or the way a concept must be built (from which pieces of knowledge and when?).

Broadly speaking, solving a design problem is both solution- and knowledge-dependent, and design is developed in accordance with the evolution of the design problem, knowledge, and solution. This study proposes a triple-helix structured model to guide the designer to think back and forth and explore satisfying solutions. The model is problem-knowledge-solution co-evolution

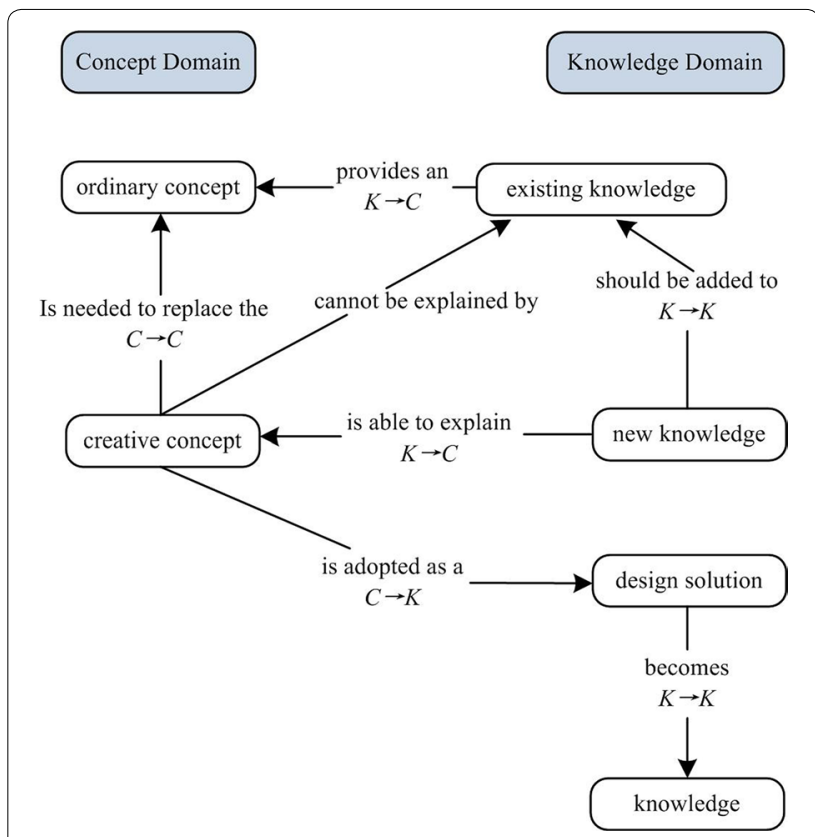

Figure 2 Illustration of C-K theory of design [34]

based, where the problem space is the direction reference for design, the knowledge space is a source of knowledge, and the solution space is an idea workshop. The model shows the changes in design thinking and leadership of the designer.

\section{Essence of Innovative Design}

To carry out an innovative design and set up its process model effectively, its essence and theoretical basis should be studied first. Innovative design is human-centered, and the design participators (i.e., the customers and designers) interact with the objects (i.e., the product, system, and process) during the design process. Innovative design is an iterative and co-evolutionary process, which can be explored and explained from three points of view of co-evolution, as illustrated in Figure 3.

First, the designer-customer-artifact co-evolution builds the relationships between the design participators and objects. Product innovation involves the creation of new concepts in product design and manufacturing to satisfy market and customer needs; one of the key drivers of product innovation is the reactions of the product to users' needs [36]. Innovative design is customer-centered, and customers are involved in product design, manufacturing, and maintenance processes. The designer takes the customers' requirements as a premise and is involved in both problem framing and solution seeking. Artifacts are designed to create certain values and significance conforming to both the customers' requirements and the natural law of product evolution. 

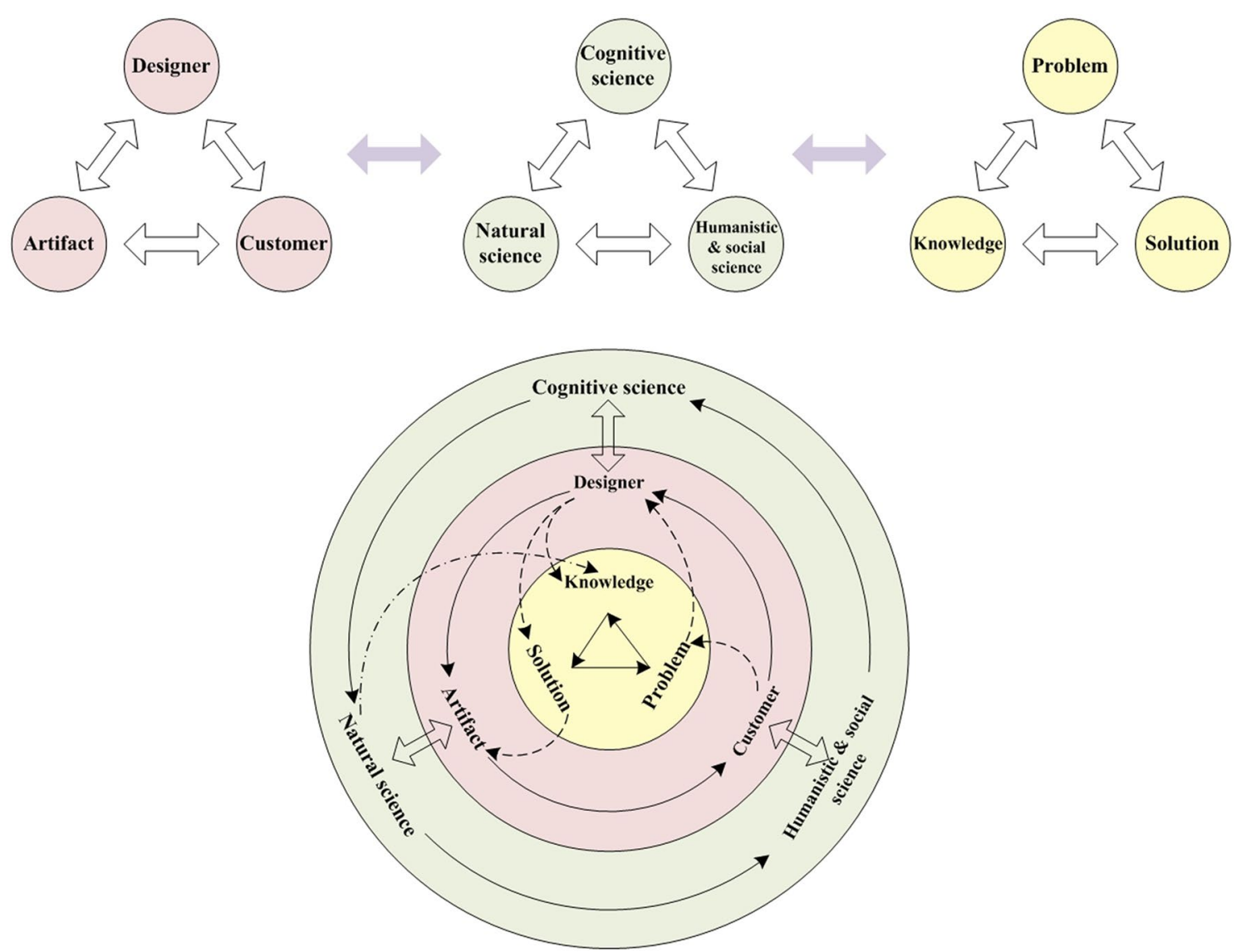

Figure 3 Essence of innovative design

Second, design knowledge expands with the co-evolution of cognitive science, humanistic and social science, and natural science knowledge. Cognitive science is used to study how designers give full play to their creativity. Humanistic and social science is used to understand the customers and design environment. Natural science is used to understand the composition and evolution of the artifacts.

Third, from the perspective of the design process, the innovative design is presented as problem-knowledgesolution co-evolution. Because the design problem is innovative with high uncertainty, the corresponding solution is in an open state. The innovative design is resolved by problem reframing, during which the original problem is refined and its representation changes. Moreover, not only the generation of new knowledge, but also the generation of general solving ideas stimulate the designer with new understanding of the problems.

The problem-knowledge-solution co-evolution builds the basic iterations of design among the participators, objects, and scientific knowledge of design. During these iterations, various scientific knowledge belonging to different domains is transferred to new design circumstances. Combined with the imagination and associative memory of the participants, the problem is gradually resolved. With the problem-knowledge-solution co-evolution, the innovative design is described as a spiraling iterative process starting from an uncertain problem, incomplete knowledge, unconstrained solution, and ending with the structured problem, acceptable solutions, and corresponding solving knowledge.

\section{Problem-Knowledge-Solution Co-evolution Based Model for Design Process}

Understanding the design problem as solution- and knowledge-dependent, and formulating a design problem often considers the design solutions and design knowledge as references. The problem, knowledge, and solution are viewed as three integral parts of design, and can be established as three evolvable spaces. As they interact with each other, the design process is pushed forward in a spiraling manner, as depicted in Figure 4.

The three evolvable spaces interact with each other through a series of mappings, forming the innovative 

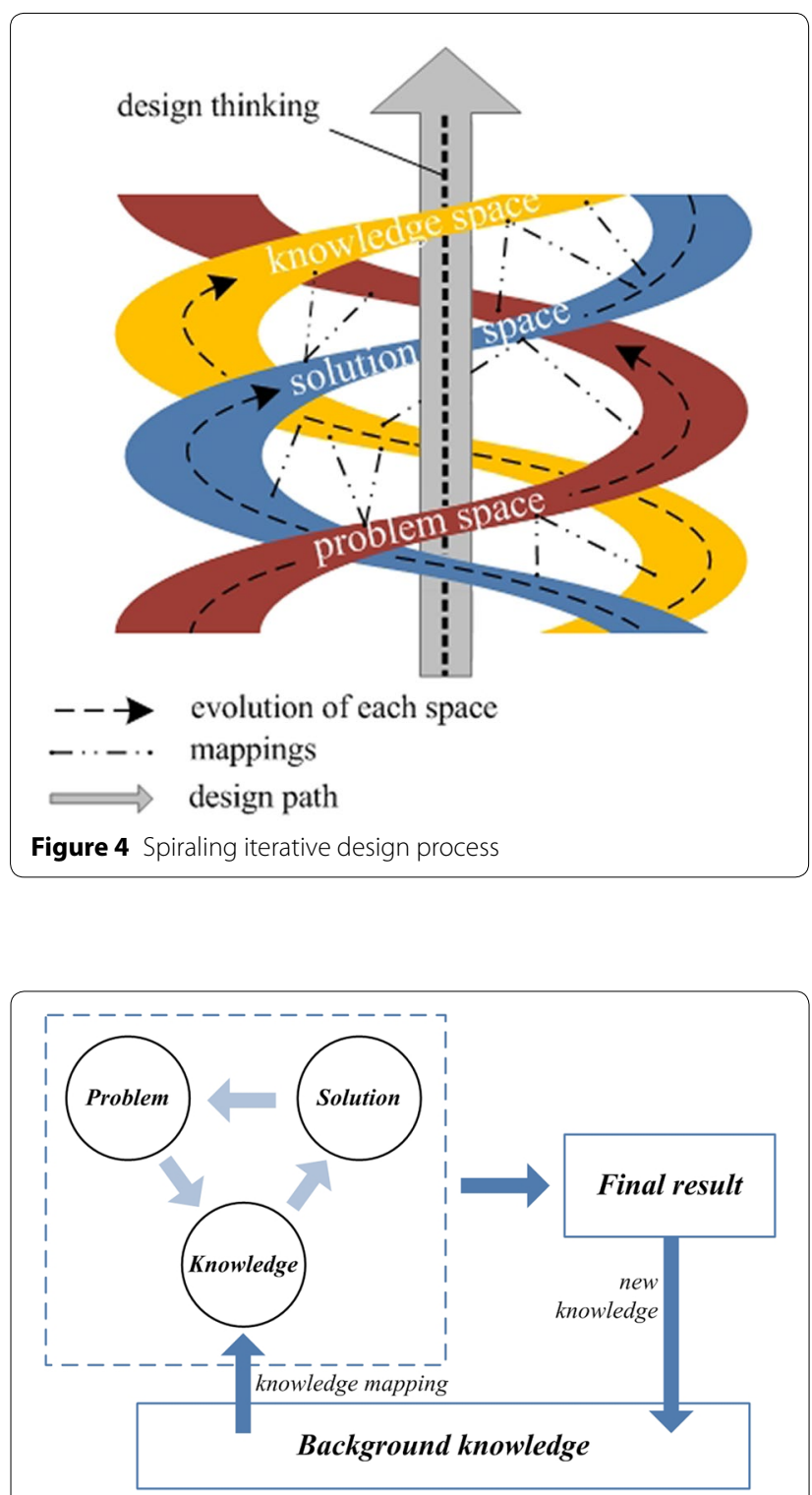

Figure 5 Problem-knowledge-solution co-evolution based model

design as a problem-knowledge-solution co-evolution shown in Figure 5. In this model, the problem presented to the designer is analyzed and resolved with the support of knowledge, which is mapped from the background knowledge and modified to be adopted for solution seeking. As the solutions are generated, they map back to the problem space to stimulate problem reframing from new perspectives. The problem-knowledge-solution co-evolution is a composite process during which the problem is clarified and resolved from fuzziness to satisfying solutions. The final result of the design is added to the background knowledge and works as the new case knowledge for future design.

\subsection{Problem Space}

The problem space (PS) is established based on the design problem presented to the designer, which is driven by the customer requirements, development of technology, or meaning of the product. The design problem received by a designer is a generalized brief, which is derived from an opportunity seeking process, existing as some form of problem statement presented to the designer. It includes some specific problems to be fixed, the customers' requirements to be achieved, or the given meanings of culture, emotion, or vision to be realized. At the very beginning of design, the initial design brief is ambiguous with less information, and is often described using variables such as design goals, requirements, and constraints.

- The design goal provides a general direction for the problem-solving process, and presents an overall statement of what the design must achieve. For example, during the design of a mobile phone, the design goal may point out that this phone can take photos.

- The requirements further define the required statements of what the design must achieve. In the example above, the requirements then further state the number of cameras and the required camera pixels.

- The constraints are the restriction factors that limit the range of the acceptable solution. In the design of the phone with a camera, the limits may be set on the cost and weight of the phone.

Because the initial design brief is ambiguous and its variables are disordered, the value of these variables is undetermined and changeable until the problem is finally defined. The problem space and its evolution are illustrated in Figure 6.

The goals and requirements of the problem are at different levels of importance and abstraction, and have their own hierarchical relationships and interconnections with each other. The requirements are stated more precisely with certain constraints, which identify the boundaries of solution searching. The set of design requirements and constraints helps in defining the problem goal with certain features. As the design continues, the understanding of the problem becomes increasingly clear through iterative analysis and reframing. Each time, the executed reframing can determine some implicit features of the problem. Consequently, the initial design brief may change, expand, contract, or be completely altered. The problem can be gradually organized as a well-defined design proposition, clarifying and constructing the design goals, requirements, and constraints of the final problem space. 


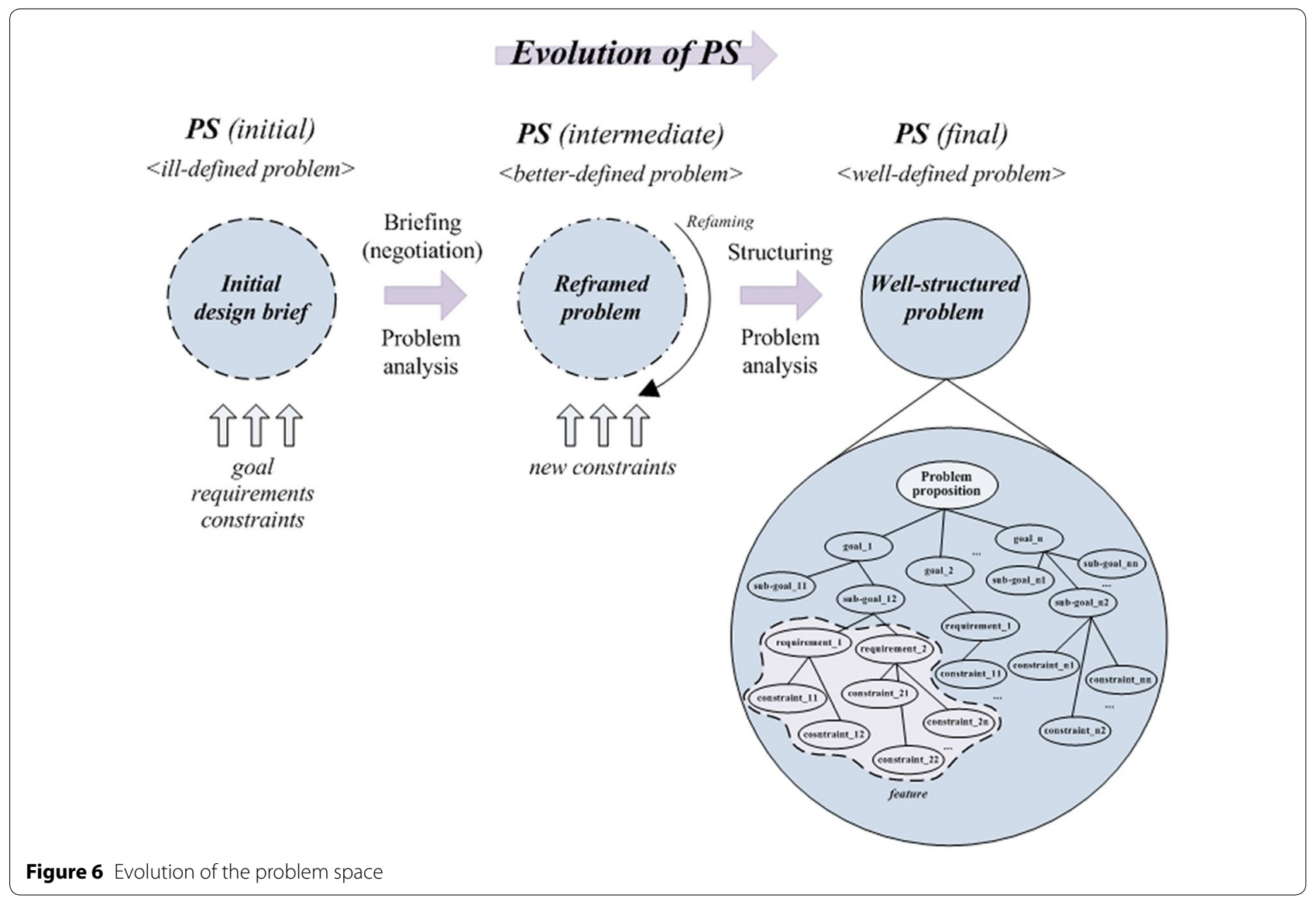

\subsection{The Knowledge Space}

Flowing along the design process, design knowledge bridges the design problem and solution, and passes useful information to support the exploration of problems and solutions, thus generate acceptable solving ideas. The knowledge space (KS) evolves as knowledge expansion, during which the target knowledge is extracted from the background knowledge and mapped to the knowledge space. The knowledge space and its evolution are shown in Figure 7.

The knowledge space contains knowledge of the designed product, whereas the background knowledge is orientated to all the knowledge accessible to designers during design. The latter can be viewed as a comprehensive database that supplies the source of pre-existing design knowledge with different abstract levels, such as inventive principles, scientific effects, patent knowledge, design cases, marketing information, etc.

Knowledge within the knowledge space is the solving knowledge adopted in the latter solution generation phase. It helps the designer generate ideas satisfying the determined requirements and constraints for pursuing the required performance. Because the designer cannot really tell what the problem defines at the beginning of the design, the initial knowledge space is an empty set and there is no prior knowledge that can be used to directly solve the problem. To solve this problem, the designer first consults the background knowledge according to the variables in the problem space, and then maps the retrieved knowledge to the knowledge space through specific operations, such as knowledge transferring, reuse, recombination, etc., to transform the knowledge from various fields into a form that can be applied to solve the current problem proposition. Based on the increasingly well-defined problem, the specificity of knowledge mapped from the background knowledge changes from abstract to concrete.

\subsection{Solution Space}

The solution space (SS) contains a set of solutions that meet the design requirements and constraints. The solution space is explored mainly through solution reasoning and evaluation processes. It evolves from an empty set to well-structured product results, which contain the final solutions and their sub-solutions. The evolution of the solution space is depicted in Figure 8.

Solving ideas are generated with the support of solving knowledge, even though the design problem has not yet 


\section{Evolution of $K S$}

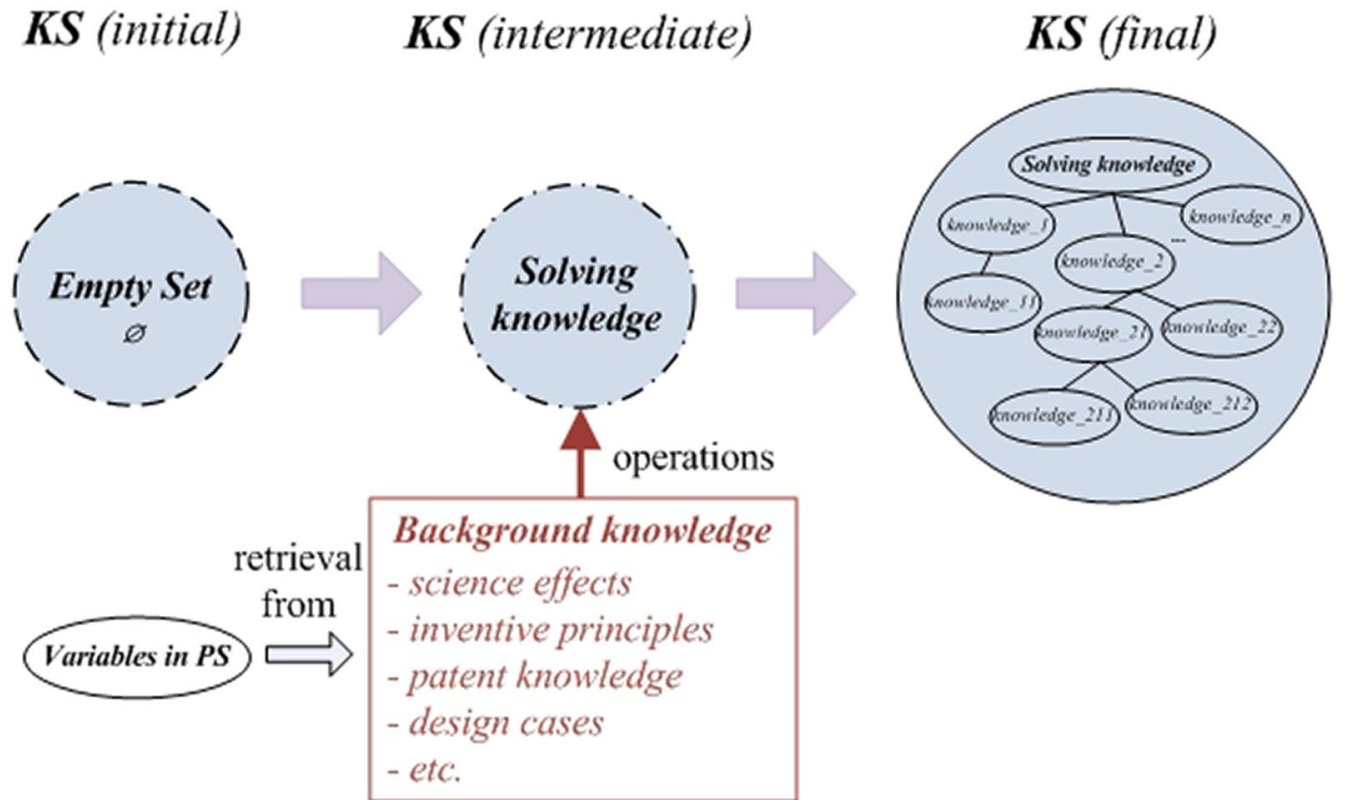

Figure 7 Evolution of the knowledge space

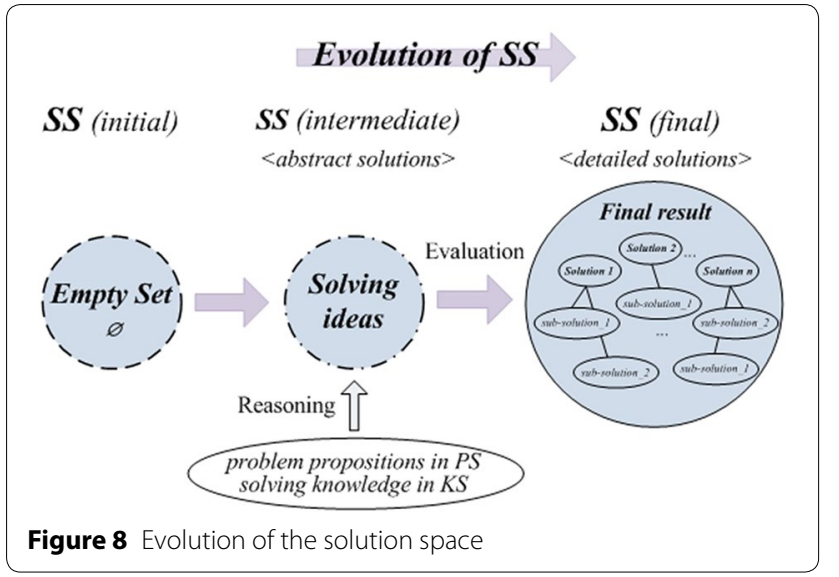

been defined precisely. To further verify their value and utility, the solving ideas should be evaluated against certain criteria derived from the design requirements and constraints. If the generated ideas are evaluated to meet the pertinent criteria, they are left in the solution space as the solution is generated. According to the ambiguous problem proposition, the designer primarily generates multiple principle solutions with varying abstract solving knowledge. As the design continues, these solutions develop under the guidance of the better-defined problem and concrete knowledge, and evolve to be more detailed and appropriate, which may be specified by some sub-solutions. In the solution space, the designer explores the satisfying solutions, but not necessarily the optimum one, leaving more possibilities for innovative design.

\section{Triple-Helix Structured Model for Product Innovative Design}

From the perspective of cognitive analysis, innovation originates from the transformation of conceptual spaces or mappings between different knowledge systems, the core of which is a mapping process transferring knowledge from one circumstance to another. During innovative design, the imaginations and associative memories of the designer are required. Innovation is often interdisciplinary, and the most creative designers can always recognize some connections that others ignore. When designers encounter an unfamiliar problem, they cannot recall some relevant operators from their memory. Consequently, the designers cannot retrieve a "best" solution from their existing knowledge system directly; therefore, the design is encountered with an impasse.

Three aspects of design need to be emphasized to break the impasse. First, the designer can reframe the problem with new representations alongside the design process from different granularity or perspectives. With the changeable representations of the problem, it can be 
understood and structured from different and interesting views, and the designer can take various paths to resolve it. Second, the designer has creative ideas based on the appropriate granularity level of the design knowledge. It is most beneficial for problem solving to use knowledge that is neither too abstract nor concise, nor too specific and detailed. Third, the retrieval scope of the background knowledge needs to be expanded so that the designer can be inspired by a wider range of solving knowledge, including both close- and far-domain knowledge.

Comprehensively considering the essence of the innovative design, the problem-knowledge-solution co-evolution, and the three ways to break the impasse of design, a triple-helix structured model is presented in this section to guide the innovative product design. It is a process model for innovative design that contains frequent iterations of analysis, synthesis, and evaluation of the problem, solution, and knowledge. With the help of this model, the designer is provided with a design strategy to plan a design project with particular design activities. The triple-helix structured model is depicted in Figure 9. The model drives the generation of design solutions through iterative mappings that carry information processing and decision-making activities across the problem, knowledge, and solution spaces.

The triple-helix structured model starts from the design problem and finally yields the design solutions. Iterative mappings facilitate the development of the design process as a circular evolution, mainly including problem structuring, knowledge expansion, and solution generation. Iterative mappings involve many connections between the abstract and detailed information of design, and show the changes and improvements made during design.

\subsection{Problem Structuring}

The problem structuring consists two kinds of design activities: problem analysis and problem reframing.

\subsubsection{Problem Analysis}

During problem analysis at the beginning of design, a set of interactions between the designer and client takes place to develop a mutual understanding of what the

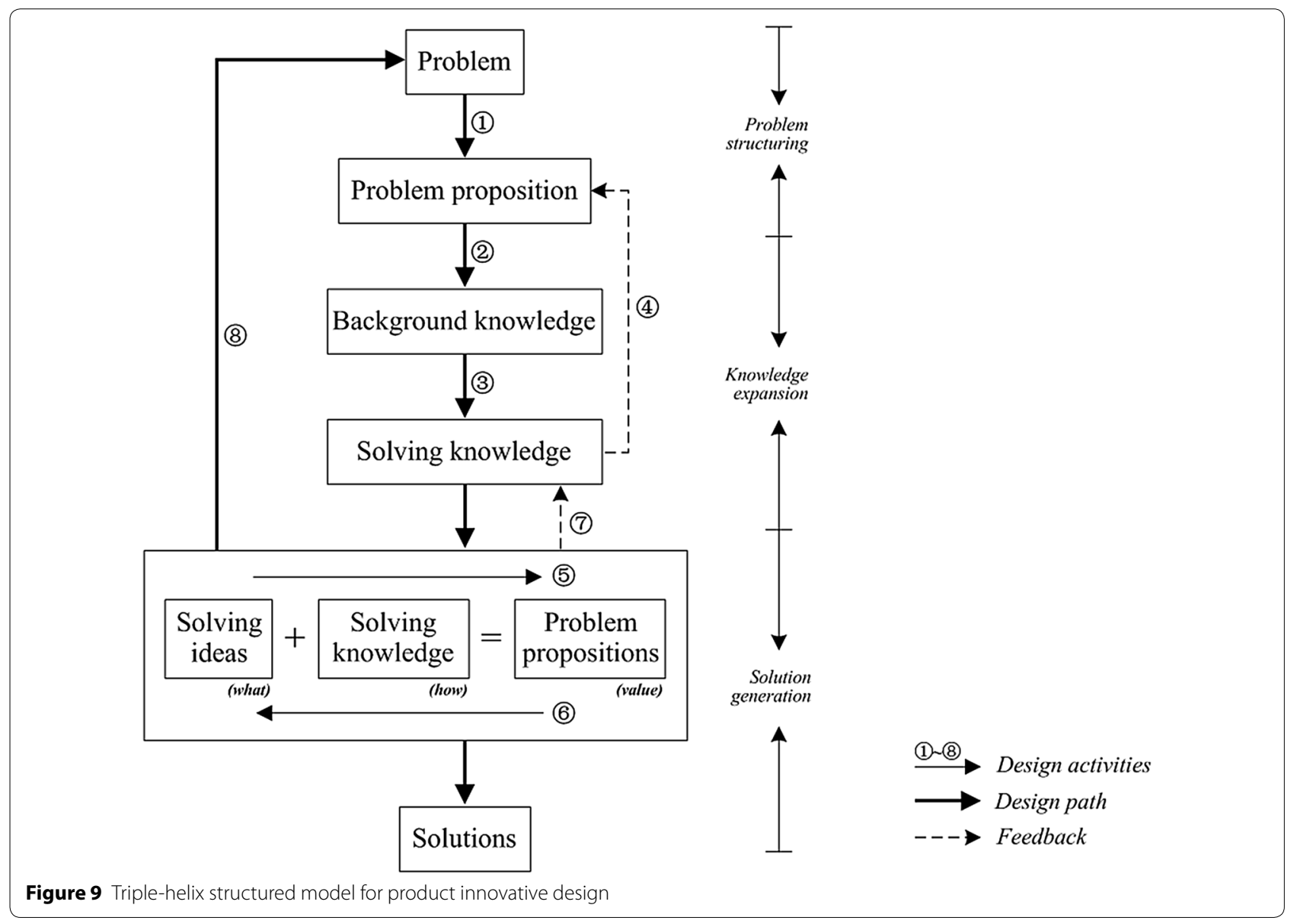


project will be about. These interactions are referred to as briefing activities.

The aim of briefing is to reframe the preliminary appreciation of the situation for both the client and designer, to create an actionable view of the project for both parties. Paton and Dorst [37] mentioned that the definitive difference between typical and innovative projects, according to the designers, was whether the project (as initially presented by the client) could be reframed during briefing.

Problem analysis aims to determine all the problem conditions and framing a final structured problem space alongside the design process as new criteria and observations are added to design. During problem analysis, the goals and requirements are transformed and represented as design propositions such as functions or other properties to be realized, providing more detailed cues for problem solving. Taking the function to be realized as an example, the product has an overall function that can be decomposed into sub-functions to clarify and detail the requirements. This decomposition process involves reasoning and decision-making to identify functional principles and carriers to meet specific goals and requirements. Meanwhile, design knowledge on the decomposition of the overall function and the reasons for making the decisions are captured, such as the kinds of functions that can be used to meet certain goals and requirements, and why function decomposition has been undertaken in a specific way.

\subsubsection{Problem Reframing}

Problem frames comprise implicit assumptions about the issues that are relevant to the problem, important values and goals, and criteria that can be used to evaluate success. Problem reframing aims at digging out all these implicit assumptions so that the variables of the problem representation and their relationships can be clarified gradually.

Reframing is assisted by abstracted co-exploring, and conjectured views of the problem situation, including areas of uncertainty. The core of problem reframing is that the designer can understand and define the problem from different granularity or perspectives. Reframing allows designers to highlight new desirable aspects for the outcome that may not have been a part of the initial frame, or at least did not hold as much value in the initial framing of the situation. Therefore, the problem is supplemented with comprehensive definitions through multiple reframing times. The reframing of a problem is influenced by the professional knowledge and personal experience of the designer.

Problem reframing can be stimulated in two ways. On one hand, it is driven by the new observations that the designer makes during solution generation. These observations represent the realistic expectation of the solution and guide the designer to reconsider the definition of the problem from the solution perspective. On the other hand, the problem is reformed because of the variation of design constraints, as the requirements of the customer and market may change with the development of design. To this end, there are sometimes new criteria and standpoints for tackling the problem, which can be viewed as new sources of problem definition.

\subsection{Knowledge Expansion}

Knowledge space expands through knowledge retrieval and processing. The designer first consults the background knowledge to search for some target knowledge. The target knowledge is usually the prior knowledge that is used to decide the right issues for consideration, how to solve these issues, and the reasons for taking particular actions and decisions.

The target knowledge is retrieved according to the problem proposition. For instance, scientific effects are selected to help achieve the functions of the design problem. Functional knowledge can be searched for in analogy reasoning. Inter-domain case knowledge is acquired for case reasoning and knowledge reuse. Principle knowledge can be retrieved to solve the contradiction between functions. After the target knowledge is selected and mapped to the knowledge space, it is then transformed and presented as solving knowledge to support the corresponding problem propositions. Compared to the target knowledge, the solving knowledge is usually interdisciplinary and fresh to the specific design domain. This transferring process creates an unknown from the known, revealing the breaking-through chances of design, identifying the connections of the design objects that have been overlooked before, and arousing design innovation.

According to the abstraction of the problem, different levels of knowledge granularity are selected from the abstract function level to the detailed case level. Selecting neither too abstract nor concise, or too specific and detailed can not only provide designers with valid solving knowledge, but also leaves them with enough room for innovation. It is affected by the individual experience and subjective judgment of the designer. Additionally, because innovation is usually associated with interdiscipline, it is demanded in the triple-helix structured model that the scope of knowledge retrieval should be expanded to provide the designer with both far- and near-domain knowledge as far as possible. Different distances of similarity knowledge can provide various stimulations for the designer, facilitating creative ideas.

The knowledge space expands under the guidance of the current problem; meanwhile, the problem is also revised with the influence of the knowledge. The solving 
knowledge feeds back to modify the representation of the problem proposition, which facilitates further understanding of the problem situation. For example, in some similarities, the designer can find more detailed information concerning the problem requirements, which can help adjust and optimize the function analysis and decomposition of the problem.

\subsection{Solution Generation}

Solution ideas are generated through reasoning and evaluation processes, during which a number of solution ideas are generated as candidates for the final design solutions. These ideas are evaluated according to the gradually better-defined problems, and the corresponding solving knowledge, during which only the ones satisfying the evaluation criteria are kept.

During the reasoning process, a type of logical inference referred to as abductive reasoning is adopted. Abductive reasoning was proposed by Pierce, and is usually used to determine possible and reasonable explanations for a given problem and introduce new ideas into design [38-43]. As shown in Figure 9, the generation of solution ideas is reasoned backwards in the equation from the current problem, which is the "value" that needs to be achieved. Using abductive reasoning, the designer reasons out a credible, promising, or at least possible hypothesis (the "what") to explain the to-be-achieved features of the design problem with the support of the solving knowledge (the "how"). For instance, to generate feasible carriers of the specific function of a product, the solving knowledge provides a principle description for the product structure working process, and with the support of the principles, the structure of the product is reasoned out to realize the expected functions and other performance requirements. Because the ultimate goal of innovative design is not to select an optimized result but a feasible one, the designer may come up with a number of different solutions. Along with the design, these ideas are improved and modified, or even abandoned, according to the specific design criteria.

Abductive reasoning is adopted far more than just generating solution ideas. What is more important is that it drives the designer to reframe the problem from the solution perspective. Although the original problem lacks detailed information, the designer can jump into the solution space before the complete definition of the problem. As the solving ideas are generated, some realistic expectations of the design are captured, which guide the designer to further understand the real problem.

The solutions develop as the problem is reframed to well-defined, and the final solution space contains structure trees of the possible solutions, which represent the physical relationships between different components of the solutions for the product.

The evaluation process is reverse to the reasoning process. The solving ideas go through evaluation to determine whether they are relevant, useful, or good; relevance and usefulness are determined against the design requirements and constraints, whereas goodness depends on the design criteria. For example, the function carriers, structures of the product, are evaluated to determine whether their feasibility, reliability, or manufacturability can meet the specifications of the functions and design constraints. If the combination of the solution structure and solving knowledge achieves the desired function, the corresponding solution idea can be left in the solution space. Otherwise, the structure or knowledge of this idea needs modification and improvement, during which a lot of useful design information can be captured, such as the particular parameter of the structure that should be considered, improvement of its performance, and the reasons for making this specific change.

\section{Case Study}

This section aims to provide more details on the triplehelix structured model for innovative product design based on an engineering design project, that is, the pressurized water reactor (PWR) coolant flow distribution device.

The distribution uniformity of the reactor coolant directly affects the thermal and hydraulic behavior in the core, and then affects the operating limit of the nuclear power plant. Additionally, uneven flow distribution causes insufficient core cooling. The large local temperature change in the reactor core negatively affects the safe operation of the reactor. Therefore, it can be deduced that the uniform distribution of the coolant in the core is highly significant for the safety and economy of a PWR.

In the existing design of various coolant flow distribution devices, the designers generally adopted numerical simulation methods to analyze the flow distribution at the core inlet, and its influencing factors, so as to provide references for the subsequent optimization of the devices. Although numerical simulation methods can optimize the existing coolant flow distribution devices, the designers are limited by their own domain knowledge and professional experience. Therefore, to overcome the shortcomings and design bottlenecks of existing products, and help the designers jump outside the box to propose innovative design solutions, a triple-helix structured model for innovative product design is adopted in the design of a PWR coolant flow distribution device. The design process for the device is as follows. 


\section{- Problem Briefing and Analysis}

The design brief represents the incomplete and imprecise mental representation of the design. In the design of a PWR coolant flow distribution device, the customer requirement is that the product should have a good effect on the uniform distribution of the coolant in the core. Moreover, the new design solution should also perform well in reducing manufacturing costs and maintenance difficulty compared to existing products. Therefore, in the initial design brief, there exists one general design goal (good effect on uniform coolant flow distribution) and two constraints (low manufacturing cost and maintenance difficulty).

To better understand this design, the existing products and the causes of uneven coolant distribution are further analyzed. Two main reasons for the uneven distribution of the coolant flow in PWR are identified. First, according to the structural characteristics of the PWR and flow characteristics of the fluid in the reactor, the coolant flow is not mixed enough; therefore, more coolant flow is distributed at the center than at the edge of the core inlet. Second, because the lower head of the pressure vessel is mostly spherical, a large number of vortexes are generated in the lower plenum. The generated vortexes consume a lot of energy, and further cause the distribution of the coolant to be uneven.

Consequently, a consensus is reached from both groups on the understanding and assumptions of the design, including the specific requirements that the product should satisfy and the structuring of the problem frame. The problem is constructed with an overall function (uniform coolant flow distribution) and two sub-functions (full fluid mixing and vortex suppression) to satisfy the goal and requirements. The problem briefing and analysis of the PWR coolant flow distribution device design is shown in Figure 10.

\section{- Knowledge Retrieval and Processing}

The target knowledge is retrieved according to the function and sub-functions of the problem. In this case, scientific effects from the function layer and design cases and patent knowledge from the detailed case layer of the background knowledge are retrieved. For example, patent knowledge of the existing flow distribution device, such as various flow equalizing devices and vortex suppression plates, is selected to inspire the designer to create solving ideas with feasible function carriers through knowledge reuse, transfer, and patent circumvention. Additionally, case knowledge of a specific structure from the architecture and mechanics domain is transferred to the current design domain for vortex suppression.

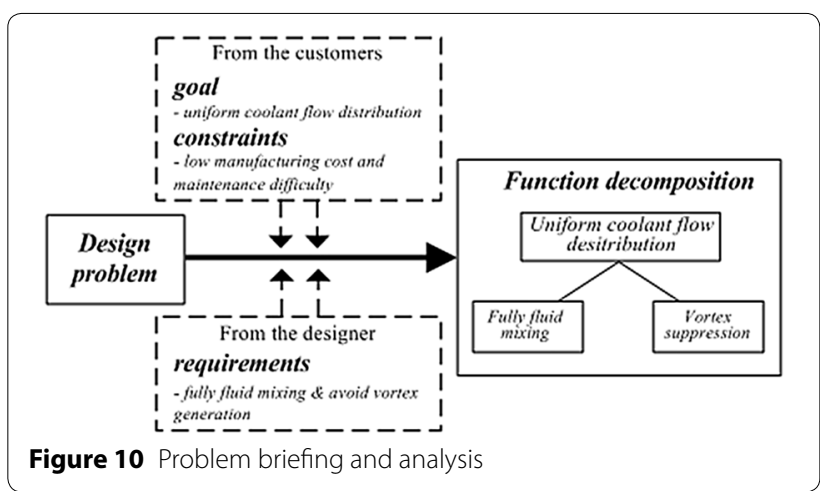

Further, while searching for the target knowledge and modifying it into the appropriate solving knowledge for the design, design issues such as technical contradictions, physical contradictions, or object-field problems, are identified within the problem proposition to block the problem-solving process. In these cases, the designer consults the corresponding background knowledge, such as the inventive principles from the TRIZ theory, to help solve these contradictions.

\section{- Solution Generation}

More than 40 function carriers are generated as potential design solutions with the support of the corresponding solving knowledge from the knowledge space. Six of the solutions proposed by the designers and their solving knowledge are listed in Table 1.

\section{- Solution Evaluation}

To evaluate the effects of the coolant flow distribution and vortex suppression of these solutions, Ansys-CFX was used for numerical simulation to calculate the normalized flow at the core inlet and obtain the velocity vector and streamline diagrams. The simulation results of each solution are compared with the results of an existing coolant flow distribution device provided by the customers. For the evaluation criterion of the uniform flow distribution, the customers require that the solutions perform better than the existing device. For the evaluation criterion of the vortex suppression, it is demanded that large vortexes should not be generated and remain in the lower plenum.

First, according to the calculation of the velocity distribution at the outlet of the lower core support plate, the overall velocity distribution of each solution can be preliminarily verified as uniform. To further describe the characteristics of the core inlet flow distribution, normalized processing is adopted. The normalized factors of 


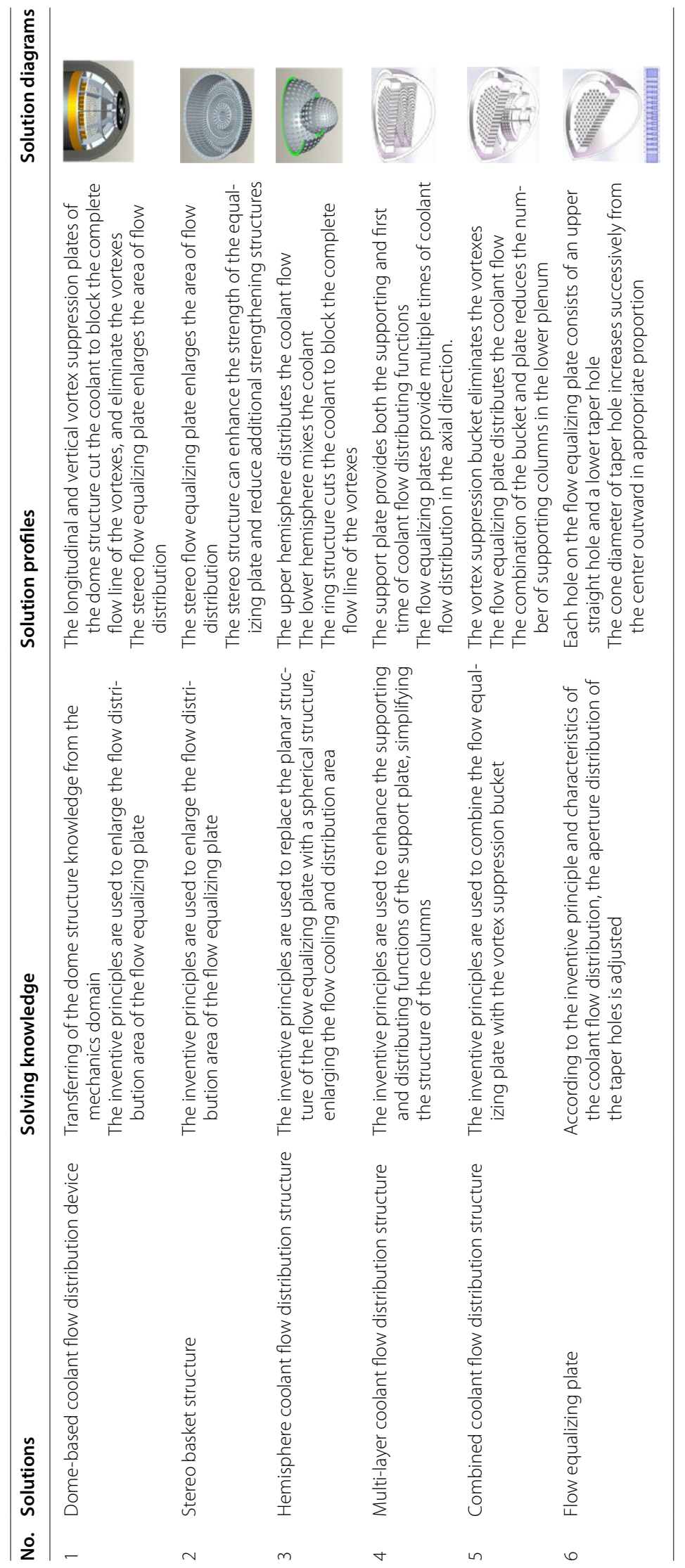


the solutions above and those of the existing device are compared to determine whether the proposed solutions satisfy the criterion of uniform flow distribution. Additionally, the velocity vector and streamline diagrams of the proposed solutions are obtained to evaluate the vortex suppression effect. The designers analyze the corresponding diagrams to determine if the solution satisfies the evaluation criterion.

Through solution evaluation, it can be observed that some of the solutions provide more frequent and uniform flow distribution of the coolant and vortex suppression in the lower plenum; these solutions can be left in the solution space as candidates for the final product results. Otherwise, the ones that do not satisfy those requirements should be modified or abandoned.

\section{- Problem Reframing}

Through solution generation, the designer proposes several solutions while also gaining an understanding of the problem from the solution perspective. Some implicit requirements and constraints are identified during the solution generation. For instance, during the numerical simulation, the designer can easily focus on the specific structure of the solution where the vortex is generated or remains. In this way, the problem is focused on the specific structure, and the designer defines the problem as fixing the unsatisfactory effect of vortex suppression. Further, the designer negotiates with customers on the solutions retained in the solution space and finds out that apart from the existing constraints, the manufacturing feasibility and supporting function of the solution structure should also be considered. Therefore, the problem proposition is reframed with new requirements and constraints.

As the problem is better defined, the designer can repeat the design process from problem analysis, knowledge retrieval and processing, to solution generation and evaluation. The initial solutions are modified or abandoned, and some new solutions may also appear. For instance, in solution 2 of Table 1, the space between the stereo flow equalizing plate and basket is reduced, which solves the problem of the generation of vortex in the original larger space. Solution 3 of Table 1 is abandoned because it cannot provide the supporting function, and may cause safety hazards if the hanging basket falls accidentally. Through the corresponding modifications, the solutions that finally meet all the design criteria can be left in the solution space and exported as the product results. The design process ends with this.

Overall, with the guidance of the triple-helix structured model for innovative product design, a number of creative solutions are generated through multiple instances of problem reframing and structural modifications. These products will then be viewed as new case knowledge of the PWR coolant flow distribution device, adding to the background knowledge for solving similar designs in the future.

\section{Conclusions}

(1) This study focused on the idea that a design problem is far more than ill-defined or wicked, but full of opportunities to innovate. The design process aimed to transfer the uncertainty behind the design problem into innovative ideas.

(2) Innovative design is an iterative and co-evolutionary process. To further explore its essence and theoretical basis, three points of view of co-evolution were proposed, namely, the designer-customerartifact co-evolution, the co-evolution of cognitive science, humanistic and social science and natural science knowledge, and the problem-knowledgesolution co-evolution.

(3) From the perspective of the design process, a triple-helix structured model based on the problemknowledge-solution co-evolution was reported. The model consists of three spaces, that is, the problem, knowledge, and solution spaces, and the mappings between them carry information processing and decision-making activities. With the help of this model and method, the designer can better understand the solving process of innovative design, leading to abstract problems, principle knowledge, and open solutions to finally evolve to the structured problem, satisfying solution, and new knowledge and design cases.

(4) The feasibility of the proposed method was validated according to a case study of a reactor coolant flow distribution device, and creative solutions were generated through multiple instances of problem reframing and structure modifications with the guidance of the triple-helix structured model for innovative product design.

\section{Acknowledgements}

Not applicable.

Authors' Contributions

$Y L$ proposed the idea of the triple-helix structured model for product innovative design; $\mathrm{QH}$ developed the model and its detailed design activities, and wrote the manuscript; YT and HWL assisted with manuscript revision and case study. All authors read and approved the final manuscript.

Authors' Information

Qian Hui, born in 1991, is currently a PhD candidate at School of Mechanical Engineering, Sichuan University, China and a member of Innovation Method and 
Creative Design Key Laboratory of Sichuan Province, China. Her research interests include innovative design.

Yan Li, born in 1954, is currently a professor at School of Mechanical Engineering, Sichuan University, China and Innovation Method and Creative Design Key Laboratory of Sichuan Province, China. His current research interests include product innovation design and optimization, numerical control technology.

Ye Tao, born in 1984, is currently an associate professor at School of Mechanical Engineering, Sichuan University, China and Innovation Method and Creative Design Key Laboratory of Sichuan Province, China. He received his doctoral degree in Mechatronic Engineering from Dalian University of Technology, China, in 2014. His research interests include product innovative design, intelligent manufacturing, and numerical control and digital manufacturing.

Hongwei Liu, born in 1992, is currently a PhD candidate at School of Mechanical Engineering, Sichuan University, China and a member of Innovation Method and Creative Design Key Laboratory of Sichuan Province, China. He received his master degree in Mechanical Engineering from Sichuan University, China, in 2017. His research interests include product innovative design.

\section{Funding}

Supported by National Natural Science Foundation of China (Grant No. 51435011).

\section{Competing Interests}

The authors declare no competing financial interests.

\section{Author Details}

${ }^{1}$ Innovation Method and Creative Design Key Laboratory of Sichuan Province, Chengdu 610065, China. ${ }^{2}$ School of Mechanical Engineering, Sichuan University, Chengdu 610065, China.

Received: 26 July 2019 Revised: 5 November 2020 Accepted: 19 November 2020

Published online: 04 December 2020

\section{References}

[1] J R Chou. An ideation method for generating new product ideas using TRIZ, concept mapping, and fuzzy linguistic evaluation techniques. Advanced Engineering Informatics, 2014, 28(4): 441-454.

[2] L Hay, A H B Duffy, C Mcteague, et al. Towards a shared ontology: A generic classification of cognitive processes in conceptual design. Design Science, 2017, 3

[3] R H Tan, Y F Dong, B J Yang, et al. Research on opportunity-driven redesign process to cooperate with training innovative engineers in China. Chinese Journal of Mechanical Engineering, 2018, 31: 75. https://doi. org/10.1186/s10033-018-0274-X.

[4] P Chuslip, Y Jin. Cognitive modeling of iteration in conceptual design. ASME 2004 International Design Engineering Technical Conferences and Computers and Information in Engineering Conference, American Society of Mechanical Engineers, 2004: 473-485.

[5] K Grace, M L Maher. Surprise and reformulation as meta-cognitive processes in creative design. Proceedings of the Third Annual Conference on Advances in Cognitive Systems, Cognitive Systems Foundation, 2015.

[6] Y Jin, P Chusilp. Study of mental iteration in different design situations. Design Studies, 2006, 27(1): 25-55.

[7] D CWynn, C M Eckert. Perspectives on iteration in design and development. Research in Engineering Design, 2017, 28(2): 153-184.

[8] Y Li, HW Liu, M D Li, et al. Review on research of design thinking. Journal of Mechanical Engineering, 2017, 53(15): 1-20. (in Chinese)

[9] L F Liu, Y Li, J L Ma, et al. Application of TRIZ in creative design based on knowledge granularity. Journal of Mechanical Engineering, 2016, 52(5): 22-32. (in Chinese)

[10] L Z Jia, C L Wu, X H Zhu, et al. Design by analogy: achieving more patentable ideas from one creative design. Chinese Journal of Mechanical Engineering, 2018, 31: 37. https://doi.org/10.1186/s10033-018-0234-5.

[11] H A Simon. The sciences of the artificial. Cambridge MA: MIT Press. 1996.
[12] M L Maher. A model of co-evolutionary design. Engineering with Computers, 2000, 16(3-4): 195-208.

[13] S Jaarsveld, T Lachmann, C Van Leeuwen. The impact of problem space on reasoning: Solving versus creating matrices. Proceedings of the Annual Meeting of the Cognitive Science Society, 2013, 35: 2632-2637.

[14] M M Welter, S Jaarsveld, T Lachmann. Problem space matters: The development of creativity and intelligence in primary school children. Creativity Research Journal, 2017, 29(2): 125-132.

[15] M L Maher. Creative design using a genetic algorithm. Computing in Civil Engineering, ASCE, 1994: 2014-2021.

[16] L Hay, A H Duffy, C McTeague, et al. Towards a shared ontology: A generic classification of cognitive processes in conceptual design. Design Science, 2017, 3.

[17] J Restrepo, H Christiaans. Problem structuring and information access in design. Journal of Design Research, 2004, 4(2): 1551-1569.

[18] C Kruger, N Cross. Solution driven versus problem driven design: strategies and outcomes. Design Studies, 2006, 27(5): 527-548.

[19] Y C Hong, I Choi. Three dimensions of reflective thinking in solving design problems: A conceptual model. Educational Technology Research and Development, 2011, 59(5): 687-710.

[20] B R Lawson. Cognitive strategies in architectural design. Ergonomics, 1979, 22(1): 59-68.

[21] P Lloyd, P Scott. Discovering the design problem. Design Studies, 1994, 15(2): 125-140.

[22] N Cross. Expertise in design: An overview. Design Studies, 2004, 25(5): 427-441.

[23] H Jiang, J S Gero, C C Yen. Exploring designing styles using a problem-solution division. Design Computing and Cognition'12, Springer, Dordrecht, 2014: 79-94.

[24] M L Maher, J Poon. Modeling design exploration as co-evolution. Computer-Aided Civil and Infrastructure Engineering, 1996, 11(3): 195-209.

[25] M Maher, H H Tang. Co-evolution as a computational and cognitive model of design. Research in Engineering Design, 2003, 14(1): 47-64.

[26] K Dorst, N Cross. Creativity in the design process: Co-evolution of problem-solution. Design Studies, 2001, 22(5): 425-437.

[27] S Wiltschnig, B T Christensen, L J Ball. Collaborative problem-solution co-evolution in creative design. Design Studies, 2013, 34(5): 515-542.

[28] A Hatchuel, B Weil. A new approach of innovative design: an introduction to CK theory. DS 31: Proceedings of ICED 03, the 14th International Conference on Engineering Design, Stockholm, 2003.

[29] A Hatchuel, B Weil. CK design theory: An advanced formulation. Research in Engineering Design, 2009, 19(4): 181.

[30] D Choulier, E Coatanéa, J Forest. The engineering design CK theory: Contributions and limits. ASME 2010 International Design Engineering Technical Conferences and Computers and Information in Engineering Conference, American Society of Mechanical Engineers, 2010: 83-92.

[31] A O Kazakci, A Hatchuel, P Le Masson, et al. Simulation of design reasoning based on CK theory: a model and an example application. DS 60: Proceedings of DESIGN 2010, the 11th International Design Conference, Dubrovnik, Croatia, 2010: 59-68.

[32] J Poelmans, G Dedene, M Snoeck, et al. An iterative requirements engineering framework based on Formal Concept Analysis and C-K theory. Expert Systems with Applications, 2012, 39(9): 8115-8135.

[33] E Kroll, P Le Masson P, B Weil. Modeling parameter analysis design moves with C-K theory. Paper presented at the International Conference on Engineering Design, ICED'13, Séoul, Korea, 2013.

[34] A S Ullah, M M Rashid, J I Tamaki. On some unique features of C-K theory of design. CIRP Journal of Manufacturing Science and Technology, 2012, 5(1): 55-66.

[35] E Coatanéa, D Choulier, J Forest. CK, an engineering design theory contributions, limits and proposals. DS 68-2: Proceedings of the 18th International Conference on Engineering Design (ICED 11), Impacting Society through Engineering Design, Vol. 2: Design Theory and Research Methodology, Lyngby/Copenhagen, Denmark, August 15-19, 2011: 323-333.

[36] Q J Peng, Y H Liu, J Zhang, et al. Personalization for massive product innovation using open architecture. Chinese Journal of Mechanical Engineering, 2018, 31: 34. https://doi.org/10.1186/s10033-018-0239-0.

[37] B Paton, K Dorst. Briefing and reframing: A situated practice. Design Studies, 2011, 32(6): 573-587. 
[38] H Takeda. Abduction for design. Formal Design Method for CAD, IFIP Transactions B-18. Elsevier Science Publishers, 1994: 221-224.

[39] G Kovács, K M Spens. Abductive reasoning in logistics research. International Journal of Physical Distribution \& Logistics Management, 2005, 35(2): 132-144.

[40] B Leavy. Design thinking - a new mental model of value innovation. Strategy \& Leadership, 2010, 38(3): 5-14.

[41] K Dorst. The core of 'design thinking' and its application. Design Studies, 2011, 32(6): 521-532.
[42] S C Y Lu, A Liu. Abductive reasoning for design synthesis. CIRP Annals, 2012,61(1): 143-146.

[43] F R Velázquez-Quesada, F Soler-Toscano, A Nepomuceno-Fernández. An epistemic and dynamic approach to abductive reasoning: Abductive problem and abductive solution. Journal of Applied Logic, 2013, 11(4): 505-522.
Submit your manuscript to a SpringerOpen ${ }^{\mathcal{O}}$ journal and benefit from:

- Convenient online submission

- Rigorous peer review

- Open access: articles freely available online

- High visibility within the field

- Retaining the copyright to your article

Submit your next manuscript at $\boldsymbol{\nabla}$ springeropen.com 